\title{
MULTIPLE CLASSIFIER SYSTEM WITH SENSITIVITY BASED DYNAMIC WEIGHTING FUSION FOR HAND GESTURE RECOGNITION
}

\author{
WENGENG HUANG ${ }^{1}$, PATRICK P. K. CHAN ${ }^{1}$, DALIN ZHOU ${ }^{2}$, YINFENG FANG ${ }^{2}$, \\ HONGHAI LIU², DANIEL S. YEUNG \\ ${ }^{1}$ School of Computer Science and Engineering, South China University of Technology, Guangzhou, China \\ ${ }^{2}$ Portsmouth University, Portsmouth, United Kingdom \\ E-MAIL: cshuangwengeng@mail.scut.edu.cn, patrickchan@scut.edu.cn, yinfeng.fang@port.ac.uk, dalin.zhou@port.ac.uk, \\ honghai.liu@icloud.com, danyeung@ieee.org
}

\begin{abstract}
:
Surface electromyogram (sEMG) signal has been applied to gesture recognition successfully. However, performance of gesture recognition degrades due to noise, which is unavoidable in practical environment. This study aims to propose a gesture recognition system using a multiple classifier system (MCS) based on random subspace. Our method makes a decision using a signal which is less sensitive to noise. Each base classifier is trained using the subset of channels selected randomly. The influence of a base classifier by a noisy sample is measured by sensitivity, defined as the perturbation of an output of a classifier. The final decision of the MCS is determined according to the output of the based stable classifiers fused by majority vote. Experimental results show that the proposed method achieves better classification performance in comparison with its base classifier and the random subspace method in terms of accuracy and robustness to noise.
\end{abstract}

\section{Keywords:}

sEMG; Robustness; Sensitivity; MCS

\section{Introduction}

A myoelectric signal, also referred to electromyogram (EMG) signal, is the electrical manifestation of a muscle contraction. EMG signal extracts information of muscle control and represent the state of a neuromuscular system [4]. EMG based gesture recognition system has been extensively studied [25-27] for prosthesis control, human-computer interaction, games etc.

Even though EMG based gesture recognition systems have achieved high accuracy, some challenging problems still remains in practice. One of them is varieties of noise which deteriorate classification performance. Major types of noise include inherent noise in the electrode movement artifact, electromagnetic noise, cross talk, internal noise, inherent instability of the signal and electrocardiographic (ECG) artifacts $[4,5]$. Unfortunately, disturbance to EMG collection is generally unpredictable in terms of time, type, and level [9] Noise is usually generated by electrode conductivity changes, sensor (electrode) location shifts, loss of electrode contact and damage to EMG electrodes [15,17-18]. White Gaussian noise (WGN) power line noise and low frequency artefact are well-known noise in EMG signal, and accuracy of recognition may be affected by WGN.

In this study, we aim to improve the robustness of EMG based gesture recognition against WGN, power line noise and low frequency artefact by adopting a Multiple Classifier System (MCS) with dynamic weighted majority vote fusion method based on sensitivity measure. Sensitivity measures how input of a classifier affect its output. Sensitivity analysis has been studies in different classifiers, such as, neural network [21-23] and SVM [24]. In the proposed system, each base classifier is trained using features extracted from different channels chosen randomly from EMG signals. A subset of the trained base classifiers id determined according to the sensitivity measure of the classifier to artificial noise on the sample. The decision is only determined by the stable classifier, i.e. the sensitive classifiers are not considered. The proposed system utilizes redundancy of EMG signal and emphasizes signals that are less sensitive to noise. Redundancy of EMG signal is useful characteristic to increase the robustness of the system. Some studies $[9,28]$ use more electrodes to increase redundancy of the signal.

The remainder of this paper is organized as follows. In Section 2, the related work on dealing with noise in EMG recording is briefly reviewed. In Section 3, our MCS with dynamic fusion based on sensitivity is introduced. Section 4 describes our experimental validation approaches and presents the experimental results. Section 5 gives the conclusions of this study. 


\section{Related work}

\subsection{Noise Reduction in EMG signal}

In EMG based gesture recognition system, EMG signal is collected by electrodes placed on the skin over a user's muscle. Then, signal preprocessing may be necessary for reducing noise and unnecessary components [5]. Feature extraction is applied to obtain the useful information from the signal [10]. Finally, the features are applied to a classifier in gesture recognition.

Many studies showed that EMG signal is sensitive to noise $[4,8,12]$. However, a noisy environment is inevitable in practice. Typical filters in signal processing are able to remove the noise with obvious frequency characteristics but not the one with random characteristics, i.e. WGN [10-11]. Examples of filters are band-pass filter and band-stop filter [5-6]. Adaptive filter [12], wavelet denoising algorithm [13], and advance digital signal filter [14] have been devised for WGN. However, WGN may not completely removed and sometimes important components of EMG signals are removed. Some studies proposed robust features [7-8] against WGN for hand gesture recognition. The performance of the gesture recognition system may still degrades when noise level is high. The methods mentioned above handle noise at preprocessing or feature extraction phase. This paper focuses on a learning model and devises a robust classifier.

\subsection{Multiple Classifier System (MCS)}

MCS [29] is composed of a number of base classifiers. Many studies show that base classifiers complement each other and make a better decision than a single classifier in many applications. Random subspace method (RSM) [19] is one of the most well-known MCS construction methods. In RSM, base classifiers are trained using different random subsets of the features. It allows the base classifiers focus on different sub-domains of the feature space. RSM works particularly well when the database provides redundant information dispersed across all features [20]. The trained base classifiers are combined by majority voting to make the final decision. RSM has been applied in many applications with satisfying results [30-32].

\section{Proposed Method}

A robust MCS model with sensitivity measure is introduced in this section. As channels of EMB signal are influenced by noise differently for a sample, a number of base classifiers trained with a dataset containing different subset of channels. Sensitivity of each classifier for an unseen sample is evaluated. A decision made by sensitive base classifiers are ignored in the final decision. We will define the sensitivity measure in Section 3.1. Section 3.2 introduces our model and its parameter is discussed in Section 3.3.

\subsection{Sensitivity Analysis}

Many sensitivity measures [1-3] have been proposed previously. We firstly define a sensitivity measure of a classifier as the change of classifier's outputs by perturbing a test sample. For a sample $x$, the sensitivity measure is defined as:

$$
\operatorname{sen}=\frac{|\{y \mid y=f(x), y=f(x+\Delta x), \Delta x \in P\}|}{|P|}
$$

where $y$ denotes the label of $x$ predicted by $f, \Delta x$ is a perturbation value and $P$ is the set containing all perturbation values. $S e n_{i}$ is large if the sample $x_{i}$ is sensitive to noise. It means that a little change on $x_{i}$ causes a huge different of the prediction.

Specifically, we generate $k$ artificial samples perturbed around a sample by changing feature values of the sample in a range $r$ for each feature. The sensitive of the sample is defined as the ratio of the number of the artificial samples classified different from the one of the original sample to the total number of the artificial samples. A larger value of the sensitive indicates a tiny change of a test sample may significantly change the output of the classifier, i.e. the classifier is sensitive to noise. The detail of calculation is showed in Algorithm 1.

Algorithm 1: Sensitivity measure of a classifier $f$ on a sample $x$

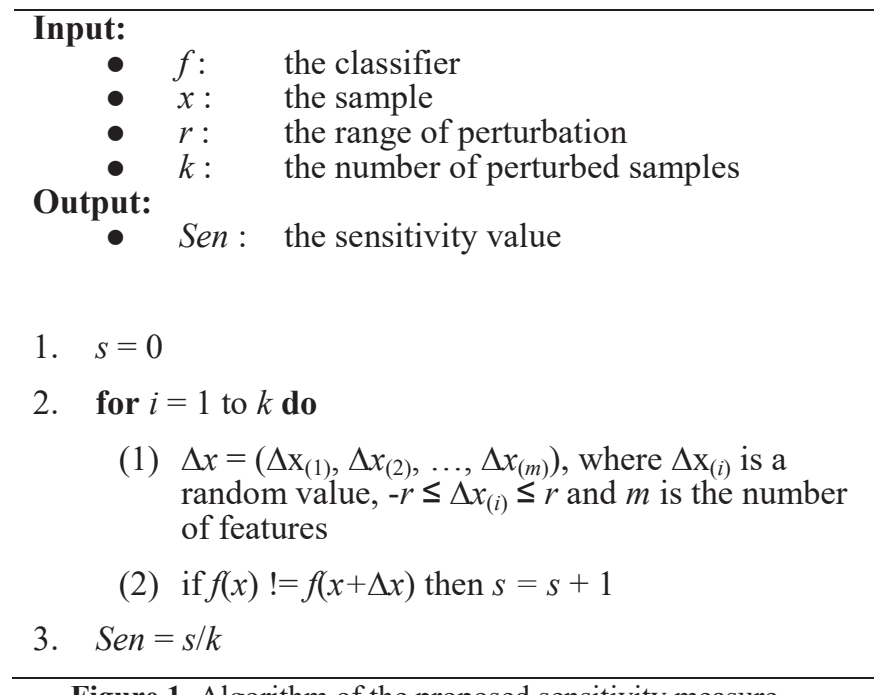

Figure 1. Algorithm of the proposed sensitivity measure 
3.2. MCS using sensitivity measure for hand gesture recognition

Given a training dataset $S_{\mathrm{C}}=\left\{\left(x_{i}, y_{i}\right) \mid i=1, \ldots, N\right\}$, where $x_{i}$ is a feature vector set extracted from the channel set (C) and $N$ is the number of samples. $y_{i}$ is the class label and $y_{i}$ $\in \mathrm{Y}$, where $\mathrm{Y}$ is the set of classes. $T$ number of base classifier is trained. The $i^{\text {th }}$ base classifiers $\left(f_{i}\right)$ is trained by using $S_{\mathrm{C}_{i}}$, where $C_{i}$ is the subset of $C$ selected randomly and $\left|C_{i}\right|=n o c$. The detailed algorithm is shown in Figure 2.

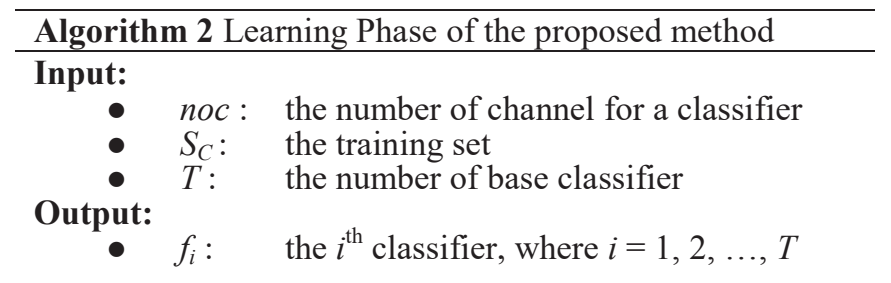

1. for $i=1$ to $T$ do

(1) Randomly select noc elements from $\mathrm{C}$ for $C_{i}$

(2) Extract $S_{\mathrm{C}_{i}}$ from $S_{\mathrm{C}}$

(3) Train $f_{i}$ using $S_{\mathrm{C}_{i}}$

Figure 2. Algorithm of training phase of the proposed method

For each test sample $x$, the sensitivity measure $\left(\operatorname{sen}_{i}\right)$ is calculated for the $i^{\text {th }}$ base classifier by (1). If $\operatorname{sen}_{i}>H$, the weight $\left(w_{i}\right)$ of $i^{\text {th }}$ base classifier is assigned to 1 ; otherwise, $w_{i}$ $=0$. The majority voting is used as a fusion method to combine the decisions of the base classifiers. The procedure of classifying a sample is given in Figure 3.

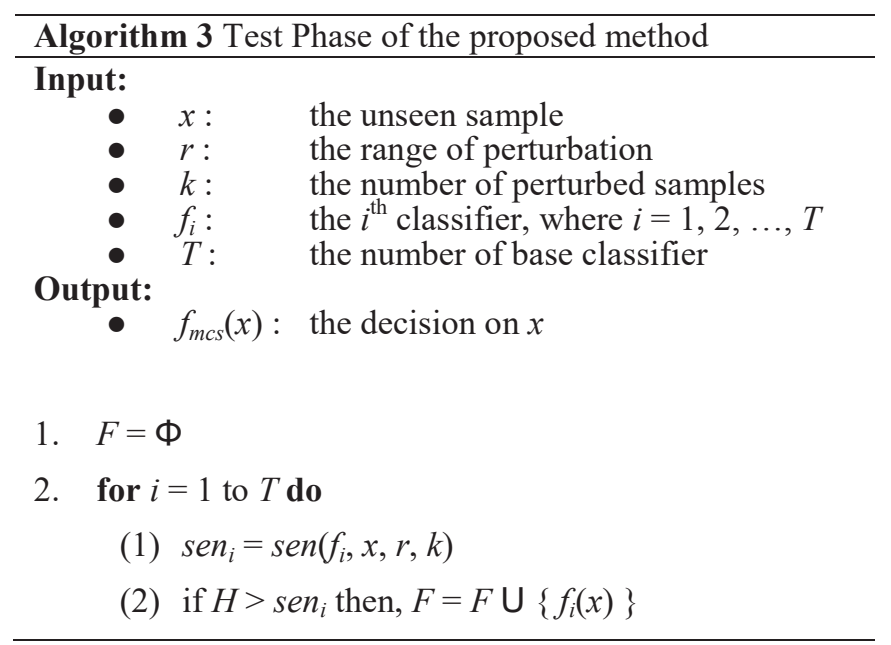

3. $f_{m c s}(x)=c$, where
$c=\arg \max _{y}(|\{f(x) \mid y=f(x), y \in Y\}, f(x) \in F|)$

Figure 3. Algorithm of training phase of the proposed method

As we assume the EMG signals we collected contain redundant information on hand gesture recognition, only subset of base classifiers can make the decision accurately. Furthermore, influence of noise on channels are different. Therefore, our method assign a small weight to a base classifier with large sensitive to noise. As a result, our method is expected to be stable and accurate since it focuses on the results of the base classifiers which are less sensitive to noise.

\subsection{Parameter Discussion}

Sensitivity measure is one of the important factors in our method. Sensitivity measure of a base classifier trained with a dataset with a small number of channels may be unstable, i.e., variance of sensitivity measure is high. Therefore, using a larger noc may reduce unreliability of sensitivity measure. On the other hand, a large noc may not be suitable as a classifier with a larger noc utilize more disturbed signal for classification. $H$ is another critical factor affects the performance significantly. Larger $H$ involves more base classifiers with large sensitive while smaller $H$ considers less base classifiers with less sensitive. Larger $T$ may learn more information about the test sample.

\section{Experiment}

\subsection{Experimental Setting}

We evaluate the performance of our proposed hand gestures recognition method by using the dataset we collected experimentally. The surface EMG signal is measured using a 16-channel sEMG acquisition system with zig electrode configuration [16]. Two right-handed university staff served as subjects. Ten hand motions are collected. Three groups of samples were collected for each subject in three days. For each group, ten hand motions were performed and each motion repeat 10 times according to the given cue signals. sEMG signals were segmented with a $300 \mathrm{~ms}$ window and $100 \mathrm{~ms}$ window shifting for feature extraction. Mean absolute value (MAV) is used for feature extraction in the experiment. There are 30 samples in one trial, 10 trials for one motion and 10 motions in total. As a result, the sample size of hand motions in one group is $30 \times 10 \times 10=3000$.

To simulate a noisy environment, WGN, $50 \mathrm{~Hz}$ power line noise and low frequency artefact are respectively added 
to $n$ number of channels selected arbitrarily. In this experiment, we set $n=4$ and 8 . Intensity of noise is set from 1000 to 6000 power of noise.

For our method, LDA is used as a base classifier. LDA and RSM are used to compare with our proposed method. Each experiment has been repeated 3 times independently. Average accuracy is measured for each method.

\subsection{Results and Discussion}

The average accuracies of the three methods without noise are shown in Table 1. Without noise, the performance of all methods are satisfying, which are higher than $99 \%$. The experimental results show that there is no significant performance difference among all methods when EMG signal without noise.

On the other hand, the experimental results of four and eight channels contaminated with WGN, power line noise and low frequency artefact are illustrated in Figures 4 and 5 respectively. These results are different from the one without noise. LDA performs worse than RSM and our methods significantly. For instance, the accuracies of LDA are lower than $4-5 \%$ and $2-3 \%$ lower than other two methods when four and eight channels with noise. This is because RSM and our method are MCS model containing base classifiers rely on feature subset. These base classifiers are not be affected by noise if they does not contain the information from the contaminated channels. In contrast, LDA is a single classifier which uses all features. Its decisions are affected by noise more significantly than others.

When the number of the noisy channels is four, the error rates of our method is lower than RSM with all intensity values. The weight of each base classifier of our method is assigned dynamically according to the sensitive measure of the base classifier on test samples. Smaller weight is assigned to a more sensitive base classifier. This setting reduces more noise influence than RSM which combines all base classifiers with even weights.

When the number of the noisy channels increase to eight, the performance difference of RSM and our method becomes smaller than the one obtained when the noisy features are four. As half of channels are contaminated, most of the base classifiers use the noisy features. Therefore, our methods may rely on small number of base classifiers, which weakens the predictive ability of our method. Therefore, it may cancel out the influence of noise to RSM and reduces the accuracy difference of both methods.

LDA has the lowest time complexity among the three methods since our model and RSM contains a number of classifiers while LDA is a single classifier. Our method has higher time complexity than RSM since the sensitivity measure of base classifiers is calculated for each testing sample.

Tabel 1. Accuracy of the three methods without noise

\begin{tabular}{|c|c|c|c|}
\hline Methods & LDA & RSM & $\begin{array}{l}\text { Our } \\
\text { method }\end{array}$ \\
\hline Accuracy & $99.73 \%$ & $99.62 \%$ & $99.57 \%$ \\
\hline
\end{tabular}

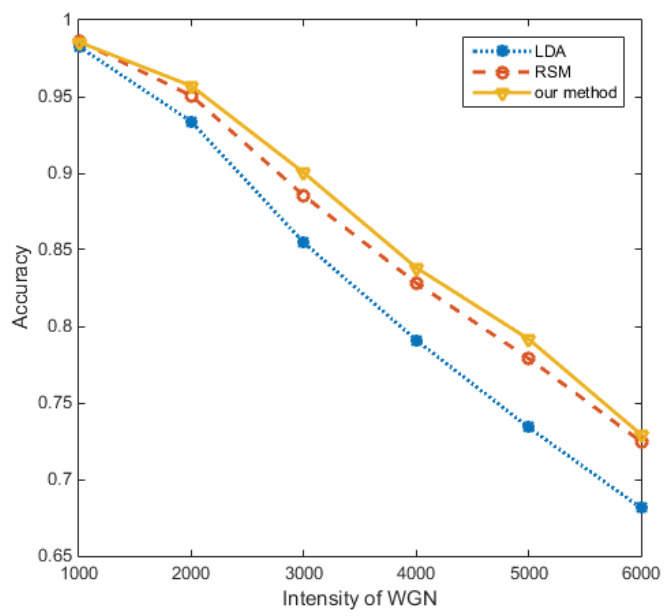

(a) WGN

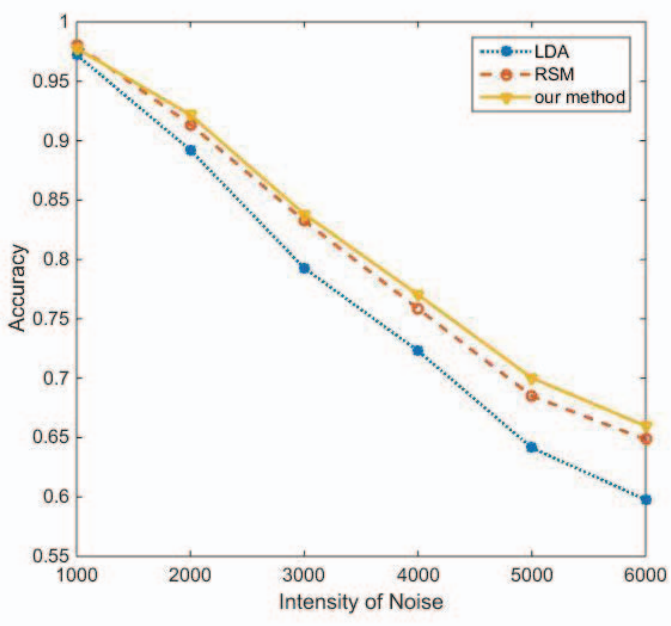

(b) Power Line Noise 


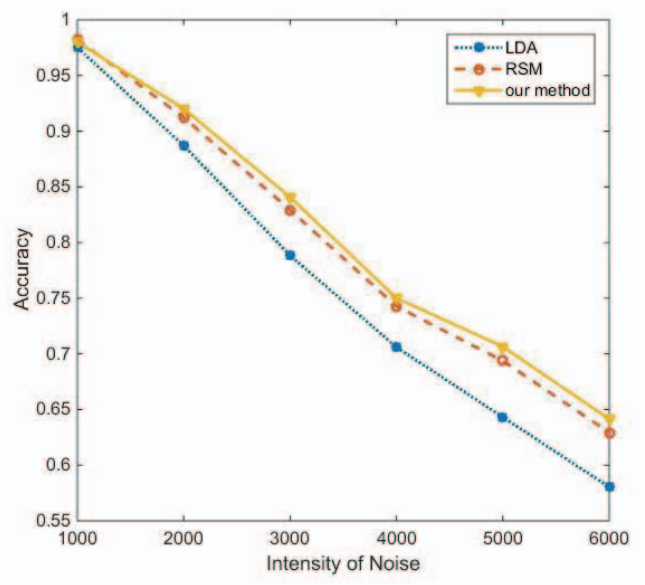

(c) Low Frequency Artefact

Figure 3. Accuracy of the three methods with four channels are contaminated by different noise

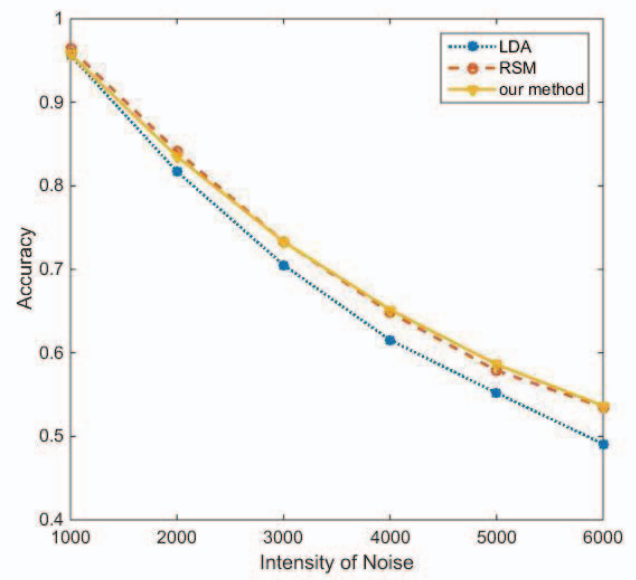

(a) WGN

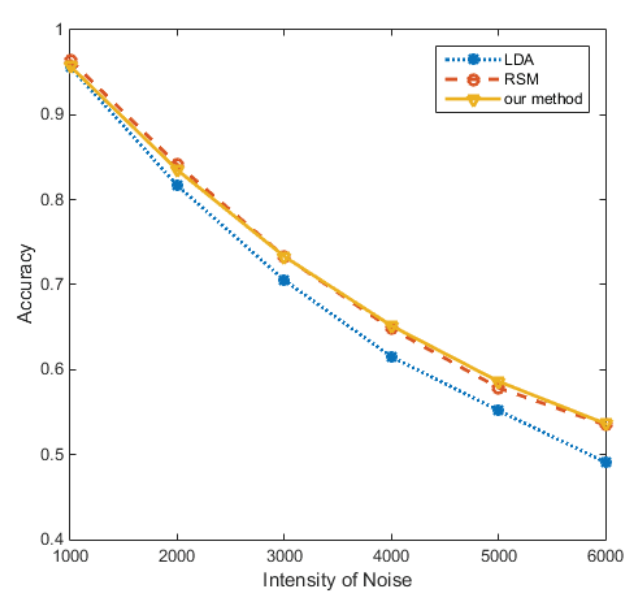

(b) Power Line Noise

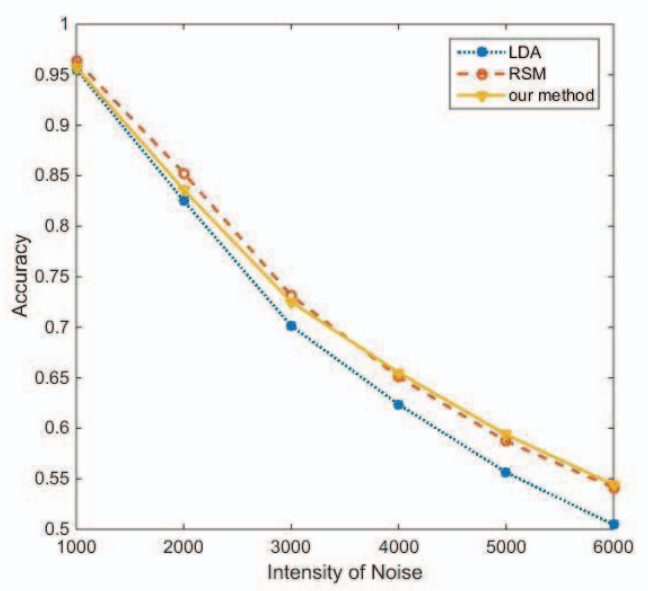

(c) Low Frequency Artefact

Figure 4. Accuracy of the three methods with eight channels are contaminated by different noise

\section{Conclusions}

This paper proposed a robust sEMG based gesture recognition method against noise. The MCS model combined a number of base classifiers trained using a subset of channels is devised. A dynamic weighted majority fusion method is used. The weight assigned to a base classifier is calculated based on sensitivity of the classifier on a test sample. The proposed system reduce the influence of noise to the hand gesture recognition by assigning a smaller weight to a sensitive base classifier. The experimental results indicate that the proposed model is more robust than LDA and RSM in the environment with WGN, power line noise and low frequency artefact. However, one drawback of our method is the time complexity since the sensitivity of each classifier should be calculated for each sample.

\section{Acknowledgements}

This work is supported by "the Fundamental Research Funds for the Central Universities" (2015ZZ092) and the National Training Program of Innovation and Entrepreneurship of China (201610561038).

\section{References}

[1] Ng, Wing WY, et al. "Empirical study on the architecture selection of RBFNN using L-GEM for multi-class problems." Machine Learning and Cybernetics (ICMLC), 2011 International Conference on. Vol. 2. IEEE, 2011.

[2] Chan, Patrick PK, Wing WY Ng, and Daniel S. Yeung. "Active 
learning using localized generalization error of candidate sample as criterion."2005 IEEE International Conference on Systems, Man and Cybernetics. Vol. 4. IEEE, 2005.

[3] Chan, Patrick PK, et al. "Dynamic fusion method using localized generalization error model." Information Sciences 217 (2012): 1-20.

[4] Chowdhury R H, Reaz M B I, Ali M A B M, et al. Surface electromyography signal processing and classification techniques[J]. Sensors, 2013, 13(9): 12431-12466.

[5] E. A. Clancy, E. L. Morin, and R. Merletti, "Sampling, Noise-reduction and Amplitude Estimation Issues in Surface Electro-myography," Journal of Electromyography and Kinesiology, vol. 12, no. 1, pp. 1-16, Feb 2002, doi:10.1016/S1050-6411(01)00033-5.

[6] M. B. I. Reaz, M. S. Hussain, and F. Mohd-Yasin, "Techniques of EMG Signal Analysis: Detection, Processing, Classification and Applications," Biological Procedures Online, vol. 8, no. 1, pp. 11-35, Mar 2006, doi:10.1251/bpo124.

[7] Phinyomark A, Limsakul C, Phukpattaranont P. EMG feature extraction for tolerance of white Gaussian noise $[\mathrm{C}] /$ Proceedings of the International Workshop and Symposium on Science and Technology. 2008: 178-183.

[8] Phinyomark A, Limsakul C, Phukpattaranont P. A novel feature extraction for robust EMG pattern recognition[J]. arXiv preprint arXiv:0912.3973, 2009.

[9] Zhang, Xiaorong, and He Huang. "A real-time, practical sensor fault-tolerant module for robust EMG pattern recognition." Journal of neuroengineering and rehabilitation 12.1 (2015): 1.

[10] M. Zardoshti-Kermani, B. C. Wheeler, K. Badie, and R. M. Hashemi, "EMG Feature Evaluation for Movement Control of Upper Extremity Prostheses," IEEE Trans. Rehabilitation Engineering, vol. 3, no. 4, pp. 324-333, Dec 1995, doi:10.1109/86.481972.

[11] Phinyomark, C. Limsakul, and P. Phukpattaranont, "A Comparative Study of Wavelet Denoising for Multifunction Myoelectric Control," Proc. Int. Conf. Computer and Automation Engineering (ICCAE '09), pp. 21-25, Mar. 2009, doi:10.1109/IC CAE.2009.57.

[12] Phinyomark, C. Limsakul, and P. Phukpattaranont, "EMG Denoising Estimation Based on Adaptive Wavelet Thresholding for Multifunction Myoelectric Control," Proc. Conf. Innovative Technologies in Intelligent Systems and Industrial Applications (CITISIA '09), pp. 171-176, July 2009,doi:10.1109/CITISIA.2009. 5224220.

[13] R. L. Ortolan, R. N. Mori, R. R. Jr. Pereira, C. M. N. Cabral, J. C. Pereira, and A. Jr. Cliquet, "Evaluation of Adaptive/Nonadaptive filtering and Wavelet Transform Techniques for Noise Reduction in EMG Mobile Acquisition Equipment," IEEE Trans. Neural Systems and Rehabilitation Engineering, vol. 11, no. 1, pp. 60-69, doi:10.1109/TNSRE.2003.810432.

[14] Lu, G., Brittain, J. S., Holland, P., Yianni, J., Green, A. L., Stein, J. F., ... \& Wang, S. (2009). Removing ECG noise from surface EMG signals using adaptive filtering. Neuroscience letters, 462(1), 14-19.

[15] Parker P, Englehart K, Hudgins B. Myoelectric signal processing for control of powered limb prostheses. J Eectromyography Kinesiol. 2006;16:541-610.

[16] Fang, Yinfeng, and Honghai Liu. "Robust sEMG electrodes configuration for pattern recognition based prosthesis control." 2014 IEEE International Conference on Systems, Man, and Cybernetics (SMC). IEEE, 2014.

[17] Sensinger JW, Lock BA, Kuiken TA. Adaptive pattern recognition of myoelectric signals: exploration of conceptual framework and practical algorithms. IEEE Trans Neural Syst Rehabil Eng. 2009;17:270-8.

[18] De Luca CJ, Donald Gilmore L, Kuznetsov M, Roy SH. Filtering the surface EMG signal: movement artifact and baseline noise contamination. J Biomech. 2010;43:1573-9.

[19] T.K. Ho, Random subspace method for constructing decision forests, IEEE Transactions on Pattern Analysis and Machine Intelligence 20 (1998) $832-844$.

[20] M. Skurichina, R. Duin, Combining Feature Subsets in Feature Selection (2005) 165 - 175.

[21] M. Stevenson, R. Winter, and B. Widrow, "Sensitivity of Feed Forward Neural Networks to Weight Errors", IEEE Trans. Neural Networks, Vol. 1, No. 1, pp. 71-80, Mar. 1990.

[22] J. Y. Choi. and C.-H. Choi, "Sensitivity analysis of multiplayer perceptron with differentiable activation functions", IEEE Trans. Neural Networks, Vol. 3, No. 1, pp. 101-107, Ian. 1992.

[23] Xiaoqin Zeng, and D. S. Yeung, "Sensitivity Analysis of Multiplayer Perceptron to Input and Weight Perturbations", IEEE Trans. Neural Networks, Vol. 12, No. 6, pp. 1358-1366, Nov. 2001

[24] Wang D F, Chan P P K, Yeung D S, et al. Feature subset selection for support vector machines through sensitivity analysis[C]//Machine Learning and Cybernetics, 2004. Proceedings of 2004 International Conference on. IEEE, 2004, 7: 4257-4262.

[25] K. Englehart, B. Hudgins, P.A. Parker, A wavelet-based continuous classification scheme for multifunction myoelectric control, IEEE Trans. Biomed. Eng. 48 (3) (2001) 302-310.

[26] K. Englehart, B. Hudgins, A robust, real-time control scheme for multifunction myoelectric control, IEEE Trans. Biomed. Eng. 50 (7) (2003) 848-854.

[27] Y. Barniv, M. Aguilar, E. Hasanbelliu, Using EMG to anticipate head motion for virtual-environment applications, IEEE Trans. Biomed. Eng. 52 (6) (2005) 1078-1093.

[28] López, Natalia M., et al. "Robust EMG sensing system based on data fusion for myoelectric control of a robotic arm." Biomedical engineering online 8.1 (2009): 1.

[29] Mayr, T. R., et al. "Multiple Classifier System." (2014).

[30] Zhang, Xiaoxun, and Yunde Jia. "A linear discriminant analysis framework based on random subspace for face recognition." Pattern Recognition 40.9 (2007): 2585-2591.

[31] Kuncheva, Ludmila I., et al. "Random subspace ensembles for fMRI classification." IEEE transactions on medical imaging 29.2 (2010): 531-542.

[32] Lai, Carmen, Marcel JT Reinders, and Lodewyk Wessels. "Random subspace method for multivariate feature selection." Pattern recognition letters 27.10 (2006): 1067-1076. 